\title{
Detection of defects in natural composite materials using a thermal imaging technique
}

\begin{abstract}
Nowadays, non-destructive testing (NDT) is frequently replacing destructive techniques in determining the properties of materials. In this study, defects in Kenaf/epoxy composite materials were detected using an inyyfrared (IR) thermal imaging technique, which is one of the most practical non-destructive techniques currently applied. Kenaf bast fibres were used to fabricate composite materials with epoxy resin as a binding material. The composites were manufactured using a manual lay-up process. The thermography analysis of the IR camera were verified by optical microscope and scanning electron microscope (SEM) investigations. The defect detection accuracy of this technology is $95 \%$.
\end{abstract}

Keyword: Natural composite materials; Detection of defects; Thermal imaging technique; Non-destructive testing (NDT) 